\title{
Visualization of the educational process of motor actions and improvement of motor skills
}

\author{
Irina Tikhonova*, Christina Pigida, Larisa Zhigaylova, Alexey Bliznuk, Olga Barcho \\ Kuban State University of Physical Education, Sports and Tourism, 350015, Krasnodar, Russia
}

\begin{abstract}
In the modern learning environment visualization takes the leading place in the process of students knowledge formation based on the receiving of the constant information flow. The particular attention in the work is paid to the development and application of the techniques and means of educational material visualization. They allow to visualize perceptual images, ideas, movements, thoughts, theoretical reasoning and constructions as visual clarity. The studying process of motor actions, quality and speed of mental operations in the process of its studying, quality and speed of its reproduction depend on many parameters, characterizing the degree of symmetry-asymmetry development in the forms and functions development. The determining factor in the system of physical education and sports training of students visualization use is the feature of its implementation at each training stage. Means and methods of visualization can be modified according to the content, purpose and form of application.
\end{abstract}

\section{Introduction}

Visualization is a consequence of pedagogical patterns development, related to the active character of the educational process students, is a consequence of the visibility principle development is an integral part of the learning process, reflecting the totality of the surrounding reality human knowledge laws, provided through sensation, perception and reflection, understanding and repulse of educational information $[5,7,9]$.

During the initial use in the definition «visualization» the content of the presentation process has been invested and understanding of data in the form of an image in order to maximize their convenience, for what the giving of a visible form to an object, subject, process have been provided (that is provided in pedagogy in the implementation of the providing visibility principle), as well as to evocation of images (really existing, or created in the mind of the author). The final and systemic result of visualization was the creation of thought images (thought forms) in the student's mind and providing so-called mental visualization.

Since the beginning of the term exploitation, there have been significant changes in the understanding of physiological processes, leading to an understanding of phenomena and environmental conditions, possibilities of the surrounding reality cognition not through visual analyzers have been identified. But at the same time its essence remained unchanged. And the keyword, characterizing visualization as a process, remains perception. It is a reflection of the complex stimulus effects based on sensations of various modalities $[1,2,4,10,11,13,15,17]$.

It should be noted that visualization implementation is based on the following basic laws of the pedagogical process, among which are: reliance on the phenomenon of apperception; reliance on the rules for constructing compositions when creating and using illustrative material; ensuring high-quality perception of information by students taking into account their individual characteristics.

Visualization of information provides not only acceleration of the knowledge formation processes, but also allows to positively influence the capabilities of students respond to inquiries of the external environment and inquiries of the society flexibly and efficiently. However, the application of an informational approach to the visualization process carries certain risks, connected with the need to regulate its flow. Saturation of the human activities totality, including redundant information learning systems, requires solving a critical problem, related to the elimination of excess information noise and its composition to accelerate processes of human knowledge acquisition based on the reflection of the received information flow.

Changes in the modern world and in the education system, concerns not only the acceleration of the information flow. Information transfer methods have changed significantly, leading to new learning tools and opportunities, information flow visualization possibilities.

An important condition for the use of visualization and the means to ensure it in the physical education system and in the athlete's training system is that they are modified each stage of training both in the content, in the purpose and in the form of application.

\footnotetext{
${ }^{*}$ Corresponding author: Tikhonova_I.74@mail.ru
} 
The use of visualization as a mean, method and technology allows to solve significant problems both in general and in private components of training, provide support for modern effective education technologies.

\section{Materials and methods}

When implementing visualization in pedagogy, the following tools are used:

- technical training tools (screen-sound learning tools, including educational films, screen learning tools, computer technologies in the form of computer demonstrations, multimedia tools, etc., interactive whiteboards);

- use of special laboratory equipment.

In the process of physical education and sports training the certain unity in approaches is used and the need for the simultaneous implementation of two approaches is recognized:

- general teaching principles are taken as the basis for learning motor actions;

- there is a need to process the principles of training taking into account the features of learning motor actions and physical exercises process. They are the main and specific means of the physical education process.

\section{Results and discussion}

The dominant form of human behavior in the course of its adaptation to environmental influences is a motor activity. It is practically the only physiologically appropriate way to activate activities of the human body internal organs, and in this regard the words of the great physiologist I.M. Sechenov (1947) about that «...all the infinite variety of external manifestations of brain activity is finally reduced to only one manifestly muscular movement», are still definitely relevant. The whole variety of processes occurring in the human body, from the perception of environmental elements to the energy supply of a working body, externally reflected and becomes visible due to a movement.

Human activity is a system of actions, united by a common goal (e.g. gaming, learning, work, etc.). Human motor activity is the process of using a combination of motor actions, aimed at moving own body or its parts in space, provided by skeletal muscle contraction during metabolic activation [6].

As a process, a person's motor activity is aimed at solving a motor task, determined by the result, either a set of motor tasks, or to the creation of external and internal conditions for the optimal solution of motor tasks.

The implementation of the visualization principle requires the maximum use of the student's capabilities in the process of motor action cognition. This requires taking into account the student's ability to visual perception in general and visual perception of motor action $[3,12,16]$.

The important aspect for the learning process of motor actions is that the basis of perception is the reflex process with the formation of temporary neural connections as complex irritants.

In theoretical terms, there are 2 approaches to the determination of the visualization process:

1) visualization of information is understood as the presentation of abstract information in visual form using graphs, charts, structural diagrams, tables, maps, etc.;

2) visualization is represented as the creation of mental images in the process of cognitive activity or as the folding of mental contents into a visual image (figure).

The use of visualization as a mean, method and technology allows to solve significant problems both in general and in the private components of training, provide support for modern effective education technologies.

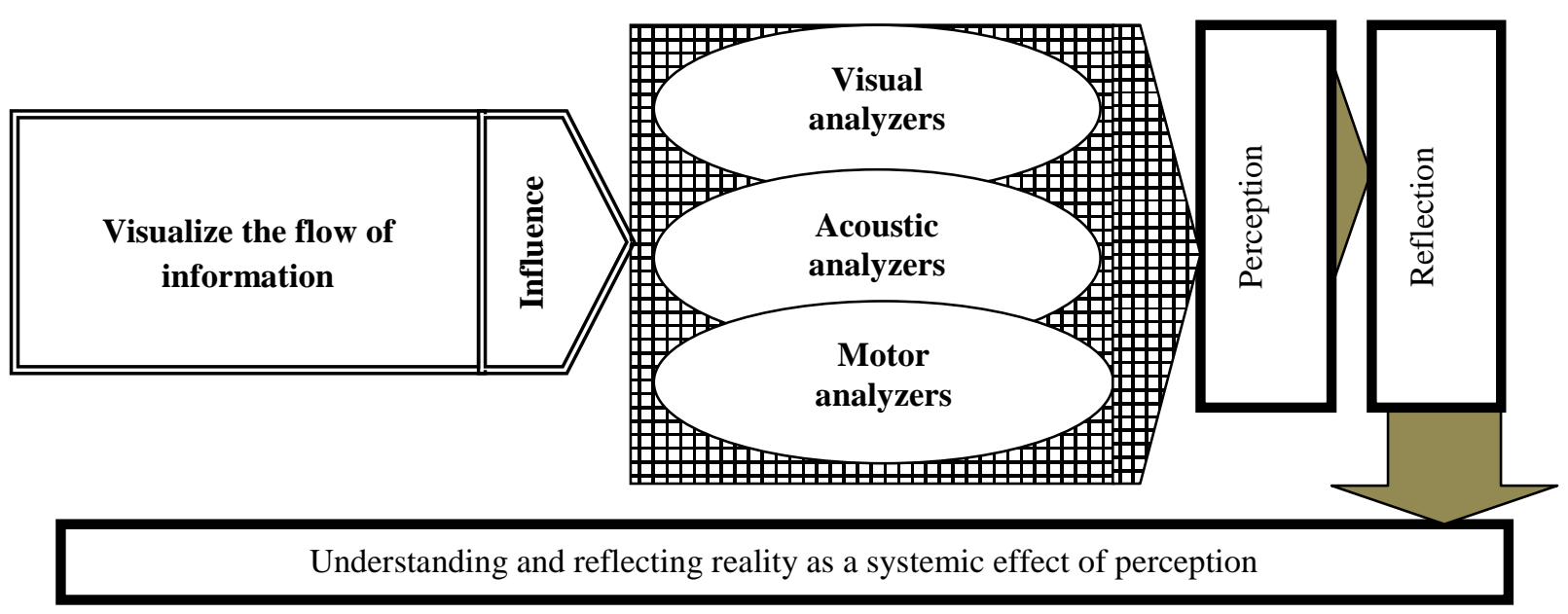

Fig. 1. Schematic picture of the visualization process

All possible types of pedagogical objects visualization operate on the principles of knowledge concentration, their generalization, the expansion of the orientation and presentation functions of visual didactic tools, algorithms of educational and cognitive actions, ensuring the process of applying visual means. In practice, many methods of 
educational material visual structuring are used, due to significant differences in nature, features and properties of various subject areas knowledge. It leads to a variety of applied methods and techniques [8, 14].

From the point of view of ensuring the learning process and identifying the conditions and direction of visualization general understanding of the physical exercise and its components technique is required. Each component of the technique requires its own level of information ownership about it and, therefore, its own nature of the visualization implementation.

Fixation of the motor action form and determining its characteristics by visualization allows to attribute the movement to a specific space, to a specific time, to determine the ways of interacting or human (by a competitor, a partner). With that such parameters are visualized, as a starting position (determining degree of readiness for execution of an action); trajectory (path of a moving body part or an object); direction of movement; range of motion (magnitude of the body parts movement path); spatio-temporal characteristics (speed); temporal characteristics (the duration of the exercises and its individual elements, individual static positions and a pace of movement); rhythm (combination in time of strong, accented parts of movement with weak, passive); qualitative characteristics of movements (parameters that are not precisely developed and not quantified, required to identify a certain qualification, but at the same time widely used to assess single movement and complex movements. They include «movement accuracy» and are determined based on the assessment of the spatial, temporal and power characteristics interaction of a motion); «movement efficiency», characterized by the absence or minimum of unnecessary movements and the minimum required energy costs; «smooth of movement», if necessary, characterized by a gradual change in muscle tension, gradual acceleration or deceleration, accuracy of a fixed trajectory reproduction when changing the direction of movement; expressiveness of movement, characterizing the expression of a mental state and emotional reflection of the execution plan.

The logical consequence of the above thoughts is the need for knowledge about the laws of the motor action cognition process general construction and understanding of the providing visualization meaning and means. Relating the above to the laws of rhythm and to the laws of symmetry and asymmetry interaction, it is necessary to understand how the quality of visual perception and the formation of a visual image are ensured at the same time and what is needed to be done to facilitate the process of mastering the motor action and moving it to the level of motor skill.

In such a way, in general terms it is provided by the activation of the information flow and provision of its effective perception, in other words, through the implementation of the visualization process stages. In this sense, visualization is the basic and determining basis for the formation of indicative activity basics and motor skills.

To determine the impact of the visualization process on the quality and motor skill formation speed, the data, obtained in the research process of the performance quality of motor actions «Sit-up» using the complex «Video Motion Analysis» and the analysis of fixed changes in articular angles, angular speed, angular accelerations of the test persons in the process of this skill formation have been matched.

The results of experimental studies confirm the importance of visualization as a principle for the formation of motor skills.

The process of motor action repetition during learning provides optimization of the execution quality by forming a motor skill, characterized as the ability of a person to perform a motor action (solve the motor problem) subject to focus on the conditions and results of the action. A characteristic feature of motor skill is that the movement is highly stable, and individual movements included in the motor action, are performed without focusing on them (movement automation). This circumstance makes the work less tiring and frees the mind for creative activity.

An important aspect for the research subject is the fact that the function of the analyzers is redistributed. The formation of a skill is connected with the increase in the role of motor and the decrease in the role of visual analyzers in the performance of an action. Visual analyzers are freed to perform the most relevant tasks for this stage, namely, ensuring control over the conditions and effectiveness of the action. From the position of the body systemic work the release of an organ from one work to perform another indicates the importance of the second factor for achieving a systemic result by the body, that is, the importance of the visualization process to ensure the high-quality implementation of the motor action.

\section{Conclusions}

Thus, it turns out that in the presence of an indicative model of movement and objective visual information about own technique, athletes of the first sports level and higher categories are able to independently determine the flaws of their own equipment and build a plan for its correction in accordance with the training tasks.

The final stage of mastering the motor action is the formation of the highest order motor skills, characterizing the ability of a person to perform a motor action (to solve the motor problem) in real life and sport activity.

Motor skill of the highest order should ensure the adaptation of the formed skill to various conditions of activity (labor, professional, sports, competitive, gaming, etc.), must correspond to the level of a person preparation for a given age or a qualification stage.

Motor skill of the highest order manifests itself in the conditions of holistic motor activity, therefore, a change in the conditions of holistic motor activity, as well as a change in the external or internal state of the action performer, should be provided by appropriate changes in the structure and quality of the movement components implementation (for example, a change in body length in ontogenesis leads to changes in the structure of running). 
The characteristic features of higher order skills are due to the presence of already formed skills and a high level of specialized knowledge. These signs, according to a number of scientists [11, 15, 17], include:

1) higher order skill, characterized by an increased role of the starting function of consciousness, moreover, only an objective analysis of the situation with a shortage of time allows to reproduce the skill that will lead to the greatest effect;

2) movements constituting specific actions, can be automated, but they may be under the control of consciousness, if correction is required in unusual conditions;

3) higher order skill always manifests itself in holistic motor activity.

The first two of the distinguished characteristic features of higher order skills are realized only when information is available and its conscious understanding, that is, during the process of motor activity visualization.

The variability of the visualization content is provided not only by the staged formation of motor skill, but also the presence of skill formation set of laws [law of uneven skill development, the law of the "plateau» (delay) in the development of skill, skill extinction law, the law of the limit absence in the development of motor skill, the law of transfer (negative or positive) skill]. Each of them requires its own approach to the implementation at the training stages, depending on the type of exercise and the preparedness of those involved.

Thus, based on the research results, it can be said that the proposed approach to training at a higher level allows to form knowledge and skills that underlie the formation of thinking strategies.

\section{References}

1. T.I. Avdeeva, M.I. Vysokos, S.I. Zykova, Bulletin of the Adygea State University. Series «Pedagogy and Psychology», 1(193), 68-71 (2017)

2. V.I. Buyanov, Young scientist, 7, 46-50 (2016)

3. D.Y. Witman, Candidate's thesis (Omsk, 2018)

4. E.V. Vorobyeva, Candidate's thesis (Moscow, 2000)

5. E.B. Ermilova, Candidate's thesis (Kazan, 1999)

6. A.Y. Zenkova, Scientific Yearbook of the Institute of Philosophy and Law, Ural Branch of the Russian Academy of Sciences, 5, 184-193 (2004)

7. A.V. Kalinichenko, Scientific and Technical Journal of Information Technologies, Mechanics, and Optics, 17(2), 359-364 (2017)

8. I.K. Latypov, Pedagogical technologies of physical education in a modern school (Pedagogical University «First of September», Moscow, 2010)

9. G.A. Nikulova, A.V. Similar, Educational Technology and Society, 2, 34-45 (2010)

10. T.I. Popova, D.V. Kolesova, Medialinguistics, 4(10), 83-94 (2015)

11. I.V. Tikhonova, The didactic principle of visualization and its implementation in physical education (Eco-invest; KGUFKST, Krasnodar, 2019)

12. I.V. Tikhonova, A.A. Bliznyuk, P.G. Omarova, Problems of modern pedagogical education, 67(3), 213-215 (2020)

13. I.V. Tikhonova, N.N. Pilyuk, O.F. Barcho, Problems of modern pedagogical education, 67(3), $215-217$ (2020)

14. A.S. Kuznetsov, Z.M. Kuznetsova, Russian Journal of Physical Education and Sport, 14(4), 5-7 (2019)

15. K.D. Chermit, S.N. Begidova, A.B. Bguashev, E.G. Verzhbitskaya, A.G. Zabolotny, Bulletin of the Adygea State University. Series «Pedagogy and Psychology», 4(146), 146-154 (2014)

16. I. Bodnar, T. Dukh, L. Hoshovska, O. Martyniv, Physical Activity Review, 6, 1-7 (2018)

17. I. Moya-Mata, L. Ruiz-Sanchis, J. Martin-Ruiz, C. Ros-Ros, Movimento, 25 (2019) 